\title{
Utility of C-Reactive Protein Levels for Early Prediction of Dengue Severity in Adults
}

\author{
Chien-Chih Chen, ${ }^{1}$ Ing-Kit Lee, ${ }^{2,3}$ Jien-Wei Liu, ${ }^{2,3}$ Shi-Yu Huang, ${ }^{1}$ and Lin Wang ${ }^{3,4}$ \\ ${ }^{1}$ Department of Emergency Medicine, Kaohsiung Chang Gung Memorial Hospital, Kaohsiung 833, Taiwan \\ ${ }^{2}$ Division of Infectious Diseases, Department of Internal Medicine, Kaohsiung Chang Gung Memorial Hospital, \\ Kaohsiung 833, Taiwan \\ ${ }^{3}$ Chang Gung University Medical College, Tao-Yuan 333, Taiwan \\ ${ }^{4}$ Department of Pediatric, Kaohsiung Chang Gung Memorial Hospital, Kaohsiung 833, Taiwan \\ Correspondence should be addressed to Ing-Kit Lee; leee@cgmh.org.tw
}

Received 4 April 2015; Revised 29 June 2015; Accepted 30 June 2015

Academic Editor: Fabrizio Montecucco

Copyright (c) 2015 Chien-Chih Chen et al. This is an open access article distributed under the Creative Commons Attribution License, which permits unrestricted use, distribution, and reproduction in any medium, provided the original work is properly cited.

\begin{abstract}
Dengue has broad clinical presentation with unpredictable clinical evolution and outcome. We aimed to evaluate the utility of C-reactive protein (CRP) levels for distinguishing between mild and severe cases in the early phase of the dengue illness. We retrospectively evaluated adults with dengue from 2006 to 2014, according to 1997 and 2009 World Health Organization (WHO) criteria for severity. Of 191 included patients, 32.9\% had nonshock dengue hemorrhagic fever (DHF), 3.1\% dengue shock syndrome (DSS), and 7.9\% severe dengue. The risk of DHF/DSS and severe dengue is significantly related to the increasing levels of CRP. Of 191 patients, 97 had CRP levels measured during the febrile (days 1-3); 85 during the critical (days 4-6); and 9 during the convalescent (days 7-10) illness phases. During the febrile phase, there was significant higher CRP level for DSS versus DF/nonshock DHF and severe dengue versus nonsevere dengue, with CRP cutoff level $30.1 \mathrm{mg} / \mathrm{L}$ (area under the receiver operating characteristic curve (AUC), $0.938 ; 100 \%$ sensitivity, $76.3 \%$ specificity) and $24.2 \mathrm{mg} / \mathrm{L}$ (AUC, $0.717 ; 70 \%$ sensitivity, $71.3 \%$ specificity), respectively. Our study highlights the utility of the CRP levels in early prediction of DSS and severe dengue in adult patients.
\end{abstract}

\section{Introduction}

Dengue is among the most significant arthropod-borne viral diseases in the world [1]. Clinically, dengue manifestation ranges from nonspecific febrile illness, dengue fever (DF), and dengue hemorrhagic fever (DHF), to the more severe form of dengue shock syndrome (DSS), according to 1997 World Health Organization (WHO) classification [2]. Limitations have been reported regarding its complexity and applicability [3, 4], and the 2009 WHO revised classification divided dengue-affected patients into two categories: nonsevere (with and without warning signs) and severe dengue [5]. The cornerstone of management of patients with dengue and prevention of dengue-related mortality is early recognition of severe cases and timely management $[6,7]$. Conversely, clinically differentiating severe and mild forms of the dengue illness is difficult during the early phase of the infection $[5,8$, 9]. Several studies have explored this question and tried using clinical and laboratory tests such as white blood cell counts, serum protein, and urea to predict severe dengue $[9,10]$. Nevertheless, dengue has broad clinical presentation with unpredictable clinical evolution and outcome. As a result, patients with dengue are often admitted for close monitoring, which can overload the health care system, particularly in resourcelimited circumstances. Warning signs (abdominal pain or tenderness, persistent vomiting, clinical fluid accumulation, mucosal bleed, lethargy or restlessness, liver enlargement $>2 \mathrm{~cm}$, and an increase in the hematocrit concurrent with a rapid decrease in the platelet count) proposed by the 2009 WHO criteria are considered as potentially key factors for early recognition of severe dengue [5]; however, the sensitivity of each warning sign in predicting severe dengue 
was reportedly extremely low [8]. A reliable and useful simple biomarker to distinguish between severe and mild illness of dengue during the early phase of infection is helpful to the treating physician in making the decision as to whether or not to admit the patient for management. C-reactive protein (CRP) is an acute-phase protein that is synthesized by the liver within six hours of the onset of inflammation [11]. Illnesses that usually cause obvious changes in CRP levels include infection, burns, trauma, inflammatory disease, and malignancy [11-13]. Clinically, CRP often used to help distinguish viral infections from bacterial infections or to monitor the response to therapy [14]. CRP has also been assessed as a biomarker to discriminate between dengue and malaria [15]. In one study, CRP levels were significantly higher in patients with DHF than in those with DF [16]. This finding showed that the degree of CRP levels might indicate the severity of dengue infection. However, there has not been a study evaluating the use of CRP levels to distinguish between mild and severe dengue during the early phase of infection. The aim of our study was to evaluate the use of CRP levels in predicting severe illness of dengue at the early phase of infection. Our findings may be valuable for clinicians working in crowded emergency rooms, particularly in resource-poor settings.

\section{Materials and Methods}

2.1. Ethics Statement. The study was approved by the Institutional Review Board of Chang Gung Memorial Hospital (CGMH) (document number 104-2250B) with a waiver of informed consent for the collection of data.

2.2. Settings and Participants. Adult patients ( $\geq 18$ years of age) with laboratory-confirmed dengue virus (DENV) infection, admitted between 2006 and 2014 at Kaohsiung CGMH, a 2,700-bed primary care and tertiary referral medical center in southern Taiwan, were included in this retrospective study. We excluded patients with dengue concurrent with bacteremia and those clinically diagnosed with superimposed bacterial infections. All patients received medical treatment at the discretion of their attending physicians. Demographic, clinical, and laboratory information of the patients was retrieved from medical records for analyses.

2.3. Categories of Dengue Illness Severity. Patients were separately categorized using clinical and laboratory data from the entire clinical course as (i) DF, nonshock DHF, or DSS, according to the 1997 WHO case classification [2], and (ii) nonsevere dengue (with and without warning signs) or severe dengue, based on the 2009 WHO case definitions [5]. Patients were diagnosed as DHF if they had fever, thrombocytopenia, bleeding, and evidence of plasma leakage (i.e., presence of hemoconcentration, pleural effusion, ascites, and/or hypoalbuminemia) [2]. DHF patients with circulatory failure were categorized as DSS [2]. Severe dengue is defined as evidence of plasma leakage associated with shock or respiratory distress, severe bleeding, or severe organ involvement [5].
2.4. Laboratory Methods. Acute and convalescent serum samples from dengue patients were obtained. Detection and amplification of DENV ribonucleic acid were performed using DENV-specific real-time reverse transcription polymerase chain reaction (RT-PCR; QuantiTect SYBR Green RTPCR Kit; QIAGEN GmbH, Hilden, Germany) as previously described [17]. The DENV-specific immunoglobulin G (IgG) antibodies were assessed by the IgG capture enzyme-linked immunosorbent assay (Panbio, Queensland, Australia) [18]. Dengue nonstructural glycoprotein-1 antigen was detected with the Platelia Dengue NS1Ag-ELISA (Bio-Rad Laboratories, Marnes-la-Coquette, France) according to the manufacturer's instructions $[19,20]$. The laboratory diagnosis of DENV infection was made based on either a positive DENVspecific RT-PCR, a positive DENV-specific nonstructural glycoprotein-1 antigen in acute-phase serum, or a fourfold increase in DENV-specific IgG antibody in convalescent serum compared with the acute phase as previously described $[6,7]$.

Patients had CRP levels measured at presentation or during hospitalization at the physician's discretion. Levels of CRP were measured by means of a highly sensitive turbidimetric immunoassay using a monoclonal antibody to CRP coated on polystyrene beads with a lower limit of detection of $0.2 \mathrm{mg} / \mathrm{L}$ (SYNCHRON CX systems) [21, 22]. A reference value of $\mathrm{CRP}<5.0 \mathrm{mg} / \mathrm{L}$ was determined in this study.

2.5. Statistical Analysis. CPR data was presented as median and range. We assessed the clinical relevance of elevated CRP level based on the severity of dengue in accordance with the 1997 and 2009 WHO dengue classification [2, 5], respectively. Further, we conducted subgroup analysis for CRP levels stratified by the febrile (days 1-3 of illness), critical (days 4-6 of illness), and convalescent (days 7-10 of illness) phases as defined by 2009 WHO criteria and compared across dengue severity classification according to 1997 and 2009 WHO criteria. Mann-Whitney $U$ test was used to determine statistical significance for continuous variables. Finally, to assess the utility of CRP levels to predict the occurrence of DSS and severe dengue, we use receiver operating characteristic curve (ROC) to determine the cutoff level of CRP for DSS and severe dengue, respectively. The area under the curve (AUC) between 0.90-1, 0.80-0.90, 0.70-0.80, $0.60-0.70$, and $0.50-0.60$ was defined as excellent, good, fair, poor, and failing, respectively. The sensitivity and specificity of the cutoff CRP level were calculated.

\section{Results}

3.1. Patient Characteristics. During the study period, 191 adult patients with dengue (mean age, 52.6 years; males, $47.6 \%)$ were included. The mean duration of illness prior to hospital presentation was $3.4( \pm 1.7)$ days. Using the 1997 WHO classification, $63.9 \%$ had DF, 32.9\% nonshock DHF, and $3.1 \%$ DSS. Using the 2009 WHO criteria, 92.1\% had nonsevere dengue and $7.9 \%$ severe dengue. Of the overall 90 DENV serotypes identified, DENV-2 accounted for $70 \%$, followed by DENV-2 (24.4\%) and DENV-1 (5.6\%). Among 
the 191 patients, the mean interval from dengue onset to blood sampling for CRP was $3.4( \pm 1.7)$ days and the mean CRP level was $19.3( \pm 27.6) \mathrm{mg} / \mathrm{L}$. Of the 191 patients, 3 DSS (severe dengue) were fatal. Demographic, clinical, and laboratory information is summarized in Table 1 .

3.2. Dengue Severity and CRP Level. For patients with DF, nonshock DHF, and DSS, median CRP levels were $8.5 \mathrm{mg} / \mathrm{L}$, $15.2 \mathrm{mg} / \mathrm{L}$, and $124.5 \mathrm{mg} / \mathrm{L}$, respectively (Table 2). The CRP level was significantly higher for nonshock DHF compared to $\mathrm{DF}(P=0.024)$, DSS compared to DF $(P<0.001)$, DSS compared to nonshock DHF $(P<0.001)$, as well as DSS compared to DF/nonshock DHF $(P=0.011)$. By the 2009 WHO dengue severity classification, median CPR levels for nonsevere dengue and severe dengue were $9.8 \mathrm{mg} / \mathrm{L}$ and $30.7 \mathrm{mg} / \mathrm{L}$, respectively (Table 3 ). When compared to patients with nonsevere dengue, patients with severe dengue had significantly higher CRP levels $(P=0.009)$.

Six patients (median age, 64.5 years [range, 18-85]; males, $50 \%$ ) had extremely high CRP levels (greater than $100 \mathrm{mg} / \mathrm{L}$ ). Of these 6 patients, 1 (16.7\%), $1(16.7 \%)$, and $4(66.7 \%)$ were classified, according to the WHO 1997 criteria, as having DF, grade 1 DHF, and DSS, respectively, whereas 5 (83.3\%) were grouped as having severe dengue based on the 2009 WHO definitions. The time from onset of dengue illness to CRP measured ranged from 1 to 5 days (median, 3 days). Among these 6 patients, acute kidney injury developed in 4 (66.7\%), severe hepatitis and gastrointestinal bleeding each developed in one $(16.7 \%)$, and $2(30 \%)$ died of DSS.

3.3. CRP Levels Stratified by Febrile, Critical, and Convalescent Phases. Of the 191 patients with dengue, 97 had CRP levels measured during their febrile phase, 85 during critical phase, and 9 during convalescent phase. By 1997 WHO dengue severity classification, the median CRP level was significantly higher for DSS versus DF $(124.5 \mathrm{mg} / \mathrm{L}$ versus $13.8 \mathrm{mg} / \mathrm{L}$; $P<0.001)$; DSS versus nonshock DHF $(124.5 \mathrm{mg} / \mathrm{L}$ versus $15.8 \mathrm{mg} / \mathrm{L} ; P=0.008)$; DSS versus $\mathrm{DF} /$ nonshock $\mathrm{DHF}$ (124.5 mg/L versus $21 \mathrm{mg} / \mathrm{L} ; P=0.001)$ in the febrile phase; and nonshock DHF versus DF $(9.9 \mathrm{mg} / \mathrm{L}$ versus $6 \mathrm{mg} / \mathrm{L}$; $P=0.016$ ) in the critical phase (Table 4). According to the 2009 WHO dengue severity classification, the median CRP level was significantly higher for severe dengue compared to nonsevere dengue during the febrile phase $(36.2 \mathrm{mg} / \mathrm{L}$ versus $14.4 \mathrm{mg} / \mathrm{L} ; P=0.025$ ) (Table 5).

3.4. The Cutoff Level of CRP to Determine DSS and Severe Dengue. The ROC curve analysis was performed for (1) DF versus nonshock DHF/DSS, (2) DF/nonshock DHF versus DSS, and (3) nonsevere dengue versus severe dengue. Regardless of the phases of illness, for DF versus nonshock DHF/DSS and DF/nonshock DHF versus DSS, the AUC was 0.652 and 0.806 , respectively. This demonstrates that CRP is insufficient to differentiate between DF and nonshock DHF/DSS. In contrast, CRP was a good discriminant between DF/nonshock DHF and DSS, with CRP cutoff level of $30.1 \mathrm{mg} / \mathrm{L}$, corresponding to $83.3 \%$ sensitivity and $81.6 \%$ specificity. In the febrile phase of illness, a CRP cutoff level of $30.1 \mathrm{mg} / \mathrm{L}$ had
TABLE 1: Patient characteristics.

\begin{tabular}{|c|c|}
\hline Variable & $N=191$ \\
\hline \multicolumn{2}{|l|}{ Demographics and comorbid conditions ${ }^{1}$} \\
\hline Mean age $( \pm S D)$, years & $52.6(16.0)$ \\
\hline Male, $n(\%)$ & $91(47.6)$ \\
\hline Type 2 diabetes mellitus, $n(\%)$ & $41(21.5)$ \\
\hline Essential hypertension, $n(\%)$ & $60(31.4)$ \\
\hline Chronic kidney disease, $n(\%)$ & $11(5.8)$ \\
\hline Previous stroke, $n(\%)$ & $5(2.1)$ \\
\hline \multicolumn{2}{|l|}{ Dengue virus serotype, $n / N(\%)$} \\
\hline Serotype 1 & $5 / 90(5.6)$ \\
\hline Serotype 2 & $63 / 90(70)$ \\
\hline Serotype 3 & $\begin{array}{l}22 / 90 \\
(24.4)\end{array}$ \\
\hline Serotype 4 & 0 \\
\hline \multicolumn{2}{|l|}{ Dengue severity, $n(\%)$} \\
\hline \multicolumn{2}{|l|}{1997 WHO classification } \\
\hline DF & $122(63.9)$ \\
\hline Nonshock DHF & $63(32.9)$ \\
\hline DSS & $6(3.1)$ \\
\hline \multicolumn{2}{|l|}{2009 WHO classification } \\
\hline Nonsevere dengue & $176(92.1)$ \\
\hline Severe dengue & $15(7.9)$ \\
\hline \multicolumn{2}{|l|}{ Clinical features at hospital presentation ${ }^{2}$} \\
\hline Mean day from onset illness to presentation $( \pm S D)$ & $3.4(1.7)$ \\
\hline Fever, $n(\%)$ & $182(95.3)$ \\
\hline Abdominal pain, $n(\%)$ & $47(24.6)$ \\
\hline Orbital pain, $n(\%)$ & $26(13.6)$ \\
\hline Bone pain, $n(\%)$ & $69(36.1)$ \\
\hline Myalgia, $n(\%)$ & $104(54.5)$ \\
\hline Headache, $n(\%)$ & $67(35.1)$ \\
\hline Rashes, $n(\%)$ & $38(20)$ \\
\hline Vomiting/nausea, $n(\%)$ & $47(24.6)$ \\
\hline Diarrhea, $n(\%)$ & $43(22.5)$ \\
\hline Petechial, $n(\%)$ & $40(20.9)$ \\
\hline Cough, $n(\%)$ & $47(24.6)$ \\
\hline Dizziness, $n(\%)$ & $43(22.5)$ \\
\hline Gastrointestinal bleeding, $n(\%)$ & $15(7.9)$ \\
\hline Gum bleeding, $n(\%)$ & $23(12)$ \\
\hline \multicolumn{2}{|l|}{ Laboratory features and outcome } \\
\hline Leukopenia $\left(\mathrm{WBC}<3.0 \times 10^{9}\right.$ cells $\left./ \mathrm{L}\right), n(\%)$ & $57(29.8)$ \\
\hline Mean hematocrit $( \pm \mathrm{SD})(\%),(n)$ & $\begin{array}{c}41 \pm 4.5 \\
(n=164)\end{array}$ \\
\hline Mean platelet count $( \pm \mathrm{SD})\left(\times 10^{9}\right.$ cells $\left./ \mathrm{L}\right),(n)$ & $\begin{array}{c}62.6 \pm 45.1 \\
(n=189)\end{array}$ \\
\hline Mean CRP $( \pm \mathrm{SD}) \mathrm{mg} / \mathrm{L}$ & $19.3(27.6)$ \\
\hline Fatality, $n(\%)$ & $3(1.6)$ \\
\hline
\end{tabular}

$\mathrm{CRP}=\mathrm{C}$-reactive protein; $\mathrm{DF}=$ dengue fever $; \mathrm{DHF}=$ dengue hemorrhagic fever; DSS = dengue shock syndrome; WBC = white blood cell count; WHO $=$ World Health Organization.

${ }^{1} \mathrm{An}$ individual patient might have more than one underlying disease/condition.

${ }^{2} \mathrm{An}$ individual patient might have more than one symptom and/or sign. 
TABLE 2: CRP level distributions by the 1997 World Health Organization dengue classification.

\begin{tabular}{lllllll}
\hline & DF $(n=122)$ & $\begin{array}{c}\text { Nonshock } \\
\text { DHF }(n=63)\end{array}$ & DSS $(n=6)$ & $P^{1}$ & $P^{2}$ & $P^{3}$ \\
\hline Median CRP (range), mg/L & $8.5(0.7-215.5)$ & $15.2(0.5-139)$ & $124.5(1.2-205.5)$ & 0.024 & $<0.001$ & $<0.001$ \\
\hline CRP = C-reactive protein; DF = dengue fever; DHF = dengue hemorrhagic fever; DSS = dengue shock syndrome. & & & \\
${ }^{1}$ DF versus nonshock DHF. \\
\end{tabular}

TABLE 3: CRP level distributions by the 2009 World Health Organization dengue classification.

\begin{tabular}{lccc}
\hline & Nonsevere dengue $(n=176)$ & Severe dengue $(n=15)$ & $P$ \\
\hline Median CRP (range), mg/L & $9.8(0.5-215.5)$ & $30.7(1.2-205.5)$ & 0.009 \\
\hline
\end{tabular}

$\mathrm{CRP}=\mathrm{C}$-reactive protein.

TABLE 4: CRP level distributions by the 1997 World Health Organization dengue classification and phase of illness.

\begin{tabular}{|c|c|c|c|c|c|c|c|}
\hline \multirow{2}{*}{ Phase of illness } & \multicolumn{3}{|c|}{ Median CRP (range)/no, mg/L } & \multirow{2}{*}{$P^{1}$} & \multirow{2}{*}{$P^{2}$} & \multirow{2}{*}{$P^{3}$} & \multirow{2}{*}{$P^{4}$} \\
\hline & DF & Nonshock DHF & DSS & & & & \\
\hline Febrile phase (days 1-3) & $13.8(0.7-56.2) / 61$ & $15.8(0.6-139.3) / 32$ & $124.5(30.7-205.5) / 4$ & 0.070 & $<0.001$ & 0.008 & 0.001 \\
\hline Critical phase (days 4-6) & $6(0.8-215.5) / 53$ & $9.9(0.5-71.1) / 31$ & $144(-) / 1$ & 0.016 & - & - & - \\
\hline Convalescent phase (days 7-10) & $3.3(1.6-10.7) / 8$ & - & $1.2(-) / 1$ & - & - & - & - \\
\hline
\end{tabular}

$\mathrm{CRP}=\mathrm{C}$-reactive protein; $\mathrm{DF}=$ dengue fever; $\mathrm{DHF}=$ dengue hemorrhagic fever; DSS = dengue shock syndrome; no = number of patients.

${ }^{1}$ DF versus nonshock DHF.

${ }^{2}$ DF versus DSS.

${ }^{3}$ Nonshock DHF versus DSS.

${ }^{4} \mathrm{DF} /$ nonshock DHF versus DSS.

TABLE 5: CRP level distributions by 2009 World Health Organization dengue classification and phase of illness.

\begin{tabular}{lccc}
\hline \multirow{2}{*}{ Phase of illness } & \multicolumn{2}{c}{ Median CRP (range)/no, mg/L } & Severe dengue \\
\hline Febrile phase (days 1-3) & Nonsevere dengue & $36.2(3.3-205.5) / 10$ & 0.025 \\
Critical phase (days 4-6) & $14.4(0.6-69) / 87$ & $29.2(6.9-144) / 4$ & 0.053 \\
Convalescent phase (days 7-10) & $8(0.5-215.5) / 81$ & $1.2(-) / 1$ & - \\
\hline
\end{tabular}

$\mathrm{CRP}=\mathrm{C}$-reactive protein; no $=$ number of patients.

100\% sensitivity and $76.3 \%$ specificity with AUC of 0.938 for differentiating between DSS and DF/nonshock DHF.

By 2009 WHO dengue severity classification, irrespective of the day of illness, the optimum CRP cutoff level was $24.2 \mathrm{mg} / \mathrm{L}$ (0.702 AUC) with $66.7 \%$ sensitivity and $76.7 \%$ specificity for nonsevere dengue versus severe dengue. In the febrile phase of illness, similarly, a CRP cutoff level of $24.2 \mathrm{mg} / \mathrm{L}$ (0.717 AUC) was obtained with $70 \%$ sensitivity and $71.3 \%$ specificity for differentiating between nonsevere dengue and severe dengue.

\section{Discussion}

CRP is used as an inflammation biomarker, particularly for bacterial infections [23, 24]. Viral infection is commonly assumed to increase CRP concentrations from 10 to $40 \mathrm{mg} / \mathrm{L}$, whereas elevated CRP $>40 \mathrm{mg} / \mathrm{L}$ is mainly found in acute bacterial infections [24-26]. In our study, median CRP level was significantly higher in those with nonshock DHF versus DF, DSS versus DF, DSS versus nonshock DHF, DSS versus $\mathrm{DF} /$ nonshock DHF, and severe dengue versus nonsevere dengue. Interestingly, the level of CRP was shown to be relatively low in DF and nonsevere dengue patients (median CRP $<10 \mathrm{mg} / \mathrm{L}$ ), whereas extremely high CRP level was found in patients with DSS (median CRP > $100 \mathrm{mg} / \mathrm{L}$ ) and severe dengue (median CRP $>30 \mathrm{mg} / \mathrm{L}$ ). The critical stage of dengue occurs most commonly towards the late febrile phase, usually after the 3rd day of illness [5]. Notably, a significant association of higher CRP levels with DSS and severe dengue was found during the first 3 days of illness (febrile phase) in our series. This finding suggests that CRP is a potentially important simple biomarker in distinguishing patients with severe form of disease from those with mild illness prior to the critical stage of dengue illness. 
Our analysis demonstrated that a CRP cutoff level of $30.1 \mathrm{mg} / \mathrm{L}$ had an excellent sensitivity (100\%) for predicting DSS, whereas a CRP cutoff level of $24.2 \mathrm{mg} / \mathrm{L}$ had a sensitivity of $70 \%$ for severe dengue, during the first 3 days of illness (febrile phase). The difference in the cutoff level of CRP for DSS and severe dengue can be explained by the different classification schemes for detecting severe cases of dengue $[2,5]$. DSS was characterized by signs of circulatory failure associated with the presence of 4 specific criteria (fever, plasma leakage, hemorrhagic manifestations, and thrombocytopenia $\left(\leq 100 \times 10^{9}\right.$ cells $\left.\left./ \mathrm{L}\right)\right)$ that met the case definition of DHF [2]. In contrast, the 2009 revised classification used a single criterion (compensated shock, severe bleeding, or severe organ involvement) for defining a severe case, independent of the presence of the 4 criteria to determine disease severity [5]. Actually, the 2009 revised classification is more sensitive in identifying severe disease, and this explains the higher numbers of cases within this group of patients when compared to DSS [27]. Our analysis suggests that using the CRP level with a cutoff level of $24 \mathrm{mg} / \mathrm{L}$ during the febrile phase of illness as a criterion for hospital admission is appropriate and that patients with high CRP levels (>30 mg/L) should receive appropriate management as those patients are at higher risk for life-threatening DSS.

In the present study, extremely high CRP $(>100 \mathrm{mg} / \mathrm{L})$ levels were noted in 6 patients, with fatal outcomes in 2. Remarkably, organ dysfunction, such as acute kidney injury and severe hepatitis, was found in a majority of patients who had extremely high CRP levels. Higher CRP levels indicate more serious tissue damage resulting from greater inflammation in the body and are often associated with poor outcome $[13,28]$. This finding emphasizes that patient with dengue with high CRP levels should therefore be intensively monitored and treated in a timely manner. Bacterial infection was excluded from our study and these findings do not apply to cases of superimposing bacterial sepsis in patients with dengue with high CRP levels.

There are some limitations to our study. First, the small number of DSS and severe dengue cases makes the statistical power quite small. Second, our study excluded patients under 18 years of age. Future prospective studies are needed to validate our findings in different populations for better generalization. Despite the limitations, this study is the first to examine the impact of single CRP testing on clinical decisionmaking and to evaluate the evidence base for the utility of this test to differentiate mild versus severe illness in adult patients with dengue.

In summary, our study highlights single measurement of CRP as a potential useful and simple biomarker to identify patients who are at risk for developing a more severe dengue illness and to help triage patients requiring hospital care. This information is especially important to clinicians in countries where medical resources are sparse and the burden of dengue is high.

\section{Conflict of Interests}

The authors declare that there is no conflict of interests regarding the publication of this paper.

\section{Authors' Contribution}

Chien-Chih Chen and Ing-Kit Lee contributed equally to this study.

\section{References}

[1] C. P. Simmons, J. J. Farrar, N. van Vinh Chau, and B. Wills, "Dengue," The New England Journal of Medicine, vol. 366, no. 15, pp. 1423-1432, 2012.

[2] World Health Organization, Dengue Hemorrhagic Fever: Diagnosis, Treatment and Control, World Health Organization, Geneva, Switzerland, 1997.

[3] J. L. Deen, E. Harris, B. Wills et al., "The WHO dengue classification and case definitions: time for a reassessment," The Lancet, vol. 368, no. 9530, pp. 170-173, 2006.

[4] S. Bandyopadhyay, L. C. S. Lum, and A. Kroeger, "Classifying dengue: a review of the difficulties in using the WHO case classification for dengue haemorrhagic fever," Tropical Medicine and International Health, vol. 11, no. 8, pp. 1238-1255, 2006.

[5] World Health Organization, Dengue: Guidelines for Diagnosis, Treatment, Prevention and Control, World Health Organization, Geneva, Switzerland, 2009.

[6] I.-K. Lee, J.-W. Liu, and K. D. Yang, "Clinical and laboratory characteristics and risk factors for fatality in elderly patients with dengue hemorrhagic fever," American Journal of Tropical Medicine and Hygiene, vol. 79, no. 2, pp. 149-153, 2008.

[7] I.-K. Lee, J.-W. Liu, and K. D. Yang, "Fatal dengue hemorrhagic fever in adults: emphasizing the evolutionary pre-fatal clinical and laboratory manifestations," PLoS Neglected Tropical Diseases, vol. 6, no. 2, Article ID e1532, 2012.

[8] T.-L. Thein, V. C. Gan, D. C. Lye, C.-F. Yung, and Y.-S. Leo, "Utilities and limitations of the World Health Organization 2009 warning signs for adult dengue severity," PLoS Neglected Tropical Diseases, vol. 7, no. 1, Article ID e2023, 2013.

[9] J. A. Potts, R. V. Gibbons, A. L. Rothman et al., "Prediction of dengue disease severity among pediatric Thai patients using early clinical laboratory indicators," PLoS Neglected Tropical Diseases, vol. 4, no. 8, article e769, 2010.

[10] V. J. Lee, D. C. Lye, Y. Sun, and Y. S. Leo, "Decision tree algorithm in deciding hospitalization for adult patients with dengue haemorrhagic fever in Singapore," Tropical Medicine and International Health, vol. 14, no. 9, pp. 1154-1159, 2009.

[11] H. Gewurz, "Biology of C-reactive protein and the acute phase response," Hospital Practice, vol. 17, no. 6, pp. 67-81, 1982.

[12] M. B. Pepys and G. M. Hirschfield, "C-reactive protein: a critical update," Journal of Clinical Investigation, vol. 111, no. 12, pp. 1805-1812, 2003.

[13] B. Clyne and J. S. Olshaker, "The C-reactive protein,” The Journal of Emergency Medicine, vol. 17, no. 6, pp. 1019-1025, 1999.

[14] D. L. Jaye and K. B. Waites, "Clinical applications of C-reactive protein in pediatrics," The Pediatric Infectious Disease Journal, vol. 16, no. 8, pp. 735-747, 1997.

[15] L. Epelboin, C. Boullé, S. Ouar-Epelboin et al., "Discriminating malaria from dengue fever in endemic areas: clinical and biological criteria, prognostic score and utility of the C-reactive protein: a retrospective matched-pair study in French Guiana," PLoS Neglected Tropical Diseases, vol. 7, no. 9, Article ID e2420, 2013.

[16] L. Á. Villar-Centeno, A. Lozano-Parra, D. Salgado-García, and Ó. F. Herrán, "Biochemical alterations as prediction markers for 
the severity of illness in dengue fever patients," Biomedica, vol. 33, no. 1, pp. 63-69, 2013.

[17] P.-Y. Shu, S.-F. Chang, Y.-C. Kuo et al., "Development of groupand serotype-specific one-step SYBR Green I-based real-time reverse transcription-PCR assay for dengue virus," Journal of Clinical Microbiology, vol. 41, no. 6, pp. 2408-2416, 2003.

[18] P.-Y. Shu, L.-K. Chen, S.-F. Chang et al., "Comparison of capture immunoglobulin $\mathrm{M}$ (IgM) and IgG enzyme-linked immunosorbent assay (ELISA) and nonstructural protein NS1 serotype-specific IgG ELISA for differentiation of primary and secondary dengue virus infections," Clinical and Diagnostic Laboratory Immunology, vol. 10, no. 4, pp. 622-630, 2003.

[19] A. Chuansumrit, W. Chaiyaratana, V. Pongthanapisith, K. Tangnararatchakit, S. Lertwongrath, and S. Yoksan, "The use of dengue nonstructural protein 1 antigen for the early diagnosis during the febrile stage in patients with dengue infection," Pediatric Infectious Disease Journal, vol. 27, no. 1, pp. 43-48, 2008.

[20] P.-Y. Shu, C.-F. Yang, J.-F. Kao et al., "Application of the dengue virus NS1 antigen rapid test for on-site detection of imported dengue cases at airports," Clinical and Vaccine Immunology, vol. 16, no. 4, pp. 589-591, 2009.

[21] S. Otsuji, H. Shibata, and M. Umeda, "Turbidimetric immunoassay of serum C-reactive protein," Clinical Chemistry, vol. 28, no. 10, pp. 2121-2124, 1982.

[22] C. P. Price, A. K. Trull, D. Berry, and E. G. Gorman, "Development and validation of a particle-enhanced turbidimetric immunoassay for C-reactive protein," Journal of Immunological Methods, vol. 99, no. 2, pp. 205-211, 1987.

[23] S. Sanders, A. Barnett, I. Correa-Velez, M. Coulthard, and J. Doust, "Systematic review of the diagnostic accuracy of Creactive protein to detect bacterial infection in non-hospitalized infants and children with fever," Journal of Pediatrics, vol. 153, no. 4, pp. 570-574.e3, 2008.

[24] R. G. Flood, J. Badik, and S. C. Aronoff, “The utility of serum Creactive protein in differentiating bacterial from non-bacterial pneumonia in children: a meta-analysis of 1230 children," The Pediatric Infectious Disease Journal, vol. 27, no. 2, pp. 95-99, 2008.

[25] D. Jupe, "The acute phase response and laboratory testing," Australian Family Physician, vol. 25, no. 3, pp. 324-329, 1996.

[26] J. Stuart and J. T. Whicher, "Tests for detecting and monitoring the acute phase response," Archives of Disease in Childhood, vol. 63 , no. 2, pp. 115-117, 1988.

[27] F. Narvaez, G. Gutierrez, M. A. Pérez et al., "Evaluation of the traditional and revised WHO classifications of dengue disease severity," PLoS Neglected Tropical Diseases, vol. 5, no. 11, Article ID e1397, 2011.

[28] C. Gabay and I. Kushner, "Acute-phase proteins and other systemic responses to inflammation," The New England Journal of Medicine, vol. 340, no. 6, pp. 448-454, 1999. 


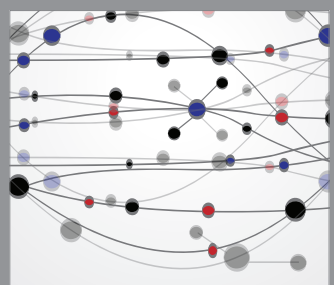

The Scientific World Journal
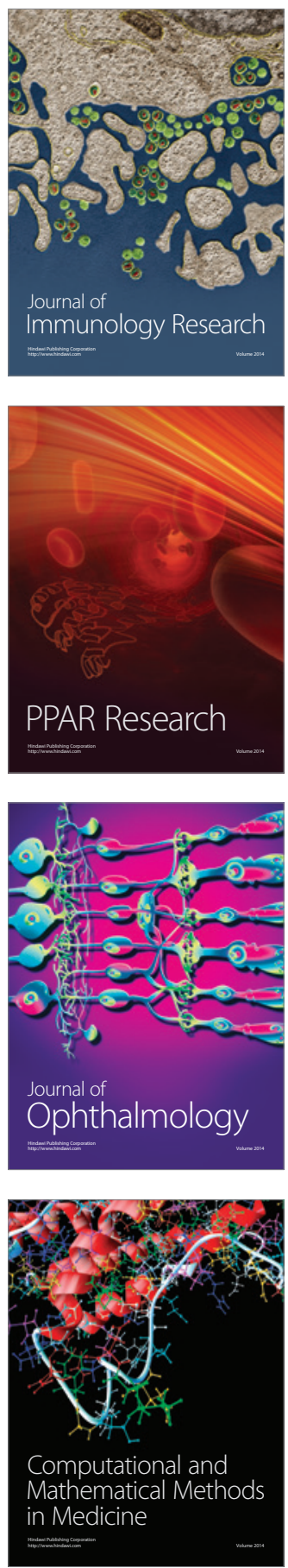

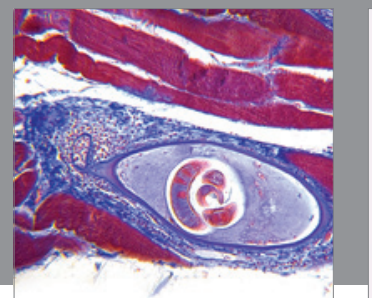

Gastroenterology

Research and Practice
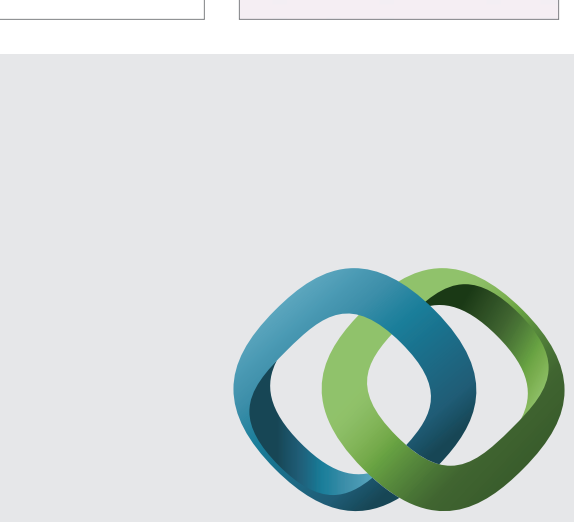

\section{Hindawi}

Submit your manuscripts at

http://www.hindawi.com
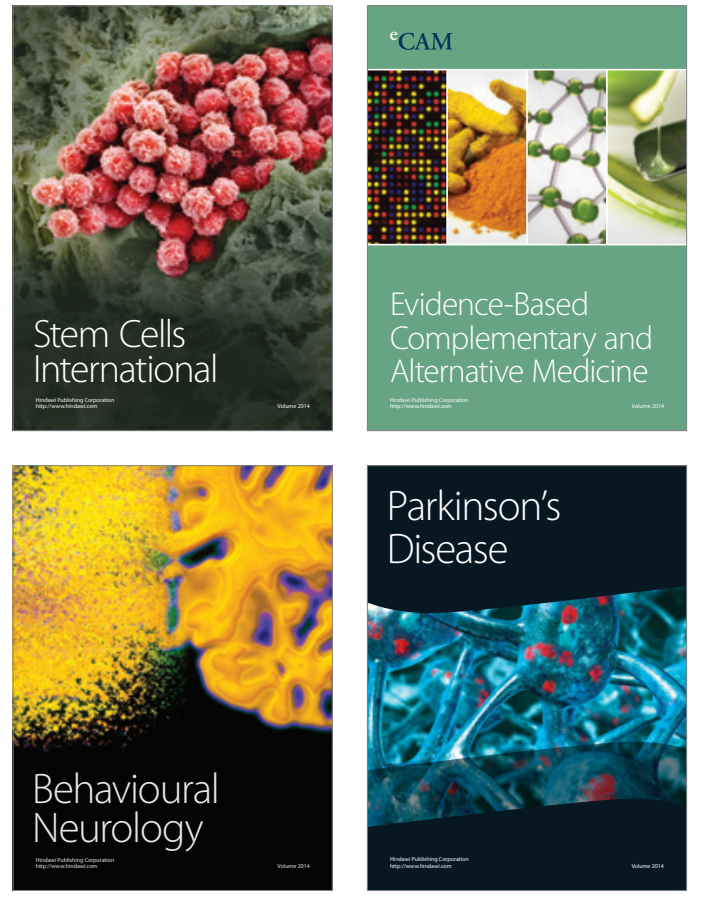
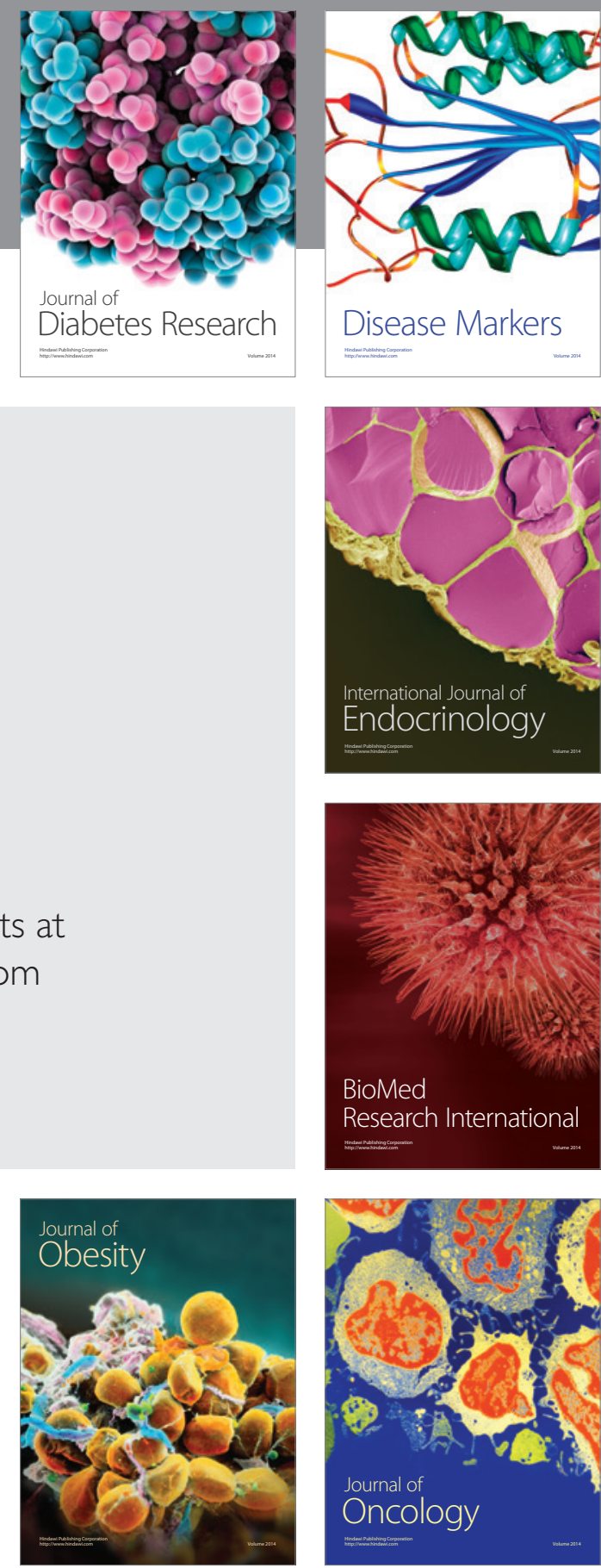

Disease Markers
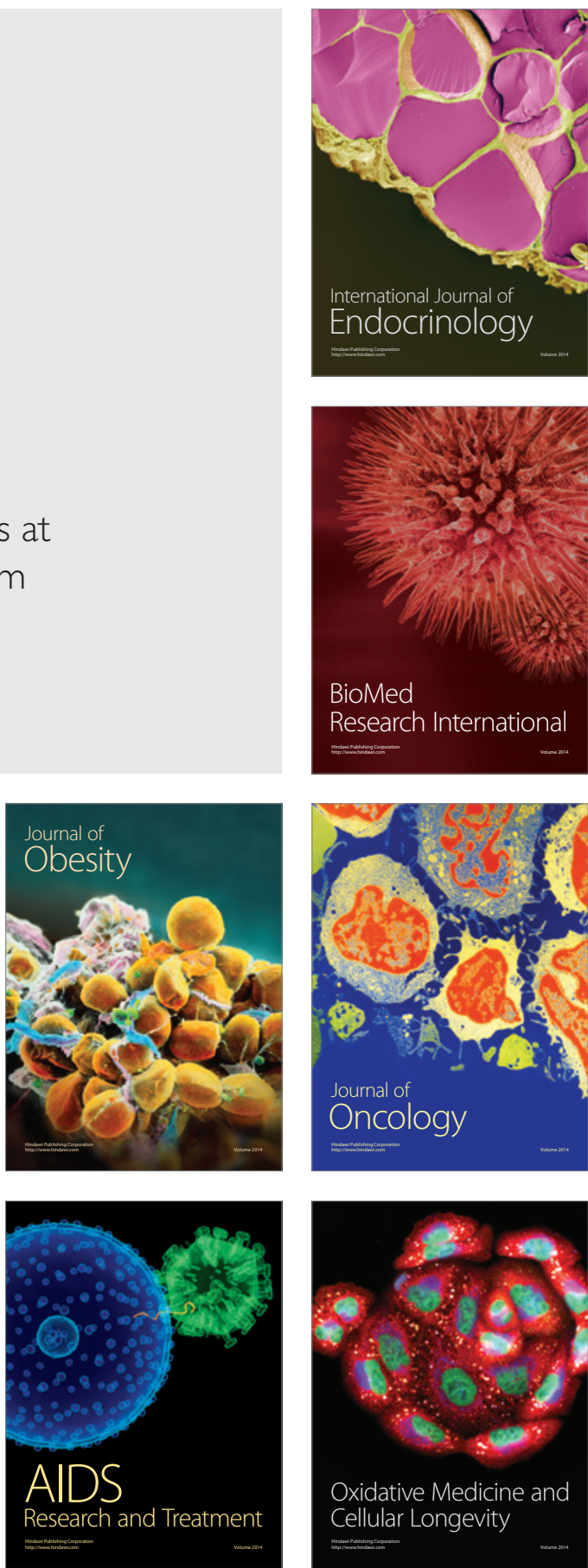